\title{
Manual of ICU Procedures
}

\author{
Mohan Gurjar (Editor). Jaypee Brothers Medical Publishers, New Delhi, India. 2016. \\ $\$ 120.50$ (CDN), 717 pages. ISBN 978-9351524229
}

\author{
Sophie Davie, MD
}

Received: 23 December 2016/Accepted: 23 January 2017/Published online: 9 March 2017

(C) Canadian Anesthesiologists' Society 2017

To manage a critically ill patient successfully, the intensive care physician must be proficient at a wide variety of procedures that allow invasive monitoring and organ support. From securing the airway, establishing vascular access, to performing therapeutic maneuvers such as thoracentesis and paracentesis, it is likely that a physician entering this specialty will require further procedural training. A book that contains explanations and guidance on a vast array of critical care procedures would be useful for any physician practicing intensive care medicine. Dr. Mohan Gurjar's Manual of ICU Procedures seeks to provide this information.

The manual is organized into five sections: airway and respiratory procedures, vascular and cardiac procedures, neurological procedures, gastrointestinal and genitourinary procedures, and a miscellaneous section. There are 61 chapters, each dedicated to a specific procedure. The chapters are presented in a similar format-a brief introduction, followed by indications and contraindications of the procedure, and a review, if applicable, of the applied anatomy. Information on both technique and equipment are then provided, followed by the necessary preparation and a step-by-step description of the procedure. The chapters end with a section on post-procedural care and complications that may arise.

The colour images are a strength of this book. Indeed, there are 428 pictures in the manual, with many chapters having a picture dedicated to each step of the procedure. Another strength of the manual is its

S. Davie, MD $(\bowtie)$

Department of Anesthesia and Perioperative Medicine,

University of Manitoba, Winnipeg, MB, Canada

e-mail: umdavie@myumanitoba.ca comprehensive nature. For example, the section on the airway not only has chapters dedicated to the obvious intensive care procedures of bag-mask ventilation and endotracheal intubation, but also has chapters on laryngeal mask airway insertion, video laryngoscopy, and fibreoptic intubation, each of which may prove useful in a difficult airway scenario. Several chapters are dedicated to intensive care unit (ICU) procedures that, although less frequently performed by critical care physicians themselves (e.g., mobilization of patients, prone positioning), are important to understand. Additionally, there are chapters on topics that one would not necessarily consider a procedure per se but are nevertheless relevant to critical care management. Examples include the chapter on transducer and pressure monitoring, which explains the physics behind the monitors. The chapter on surveillance of ICU-acquired infections is also an example of "non-procedural" ICU management.

Other chapters in this manual cover procedures that would require further specialized training. Examples include the chapters on bedside laparoscopy and dynamic abdominal wall closure for the open abdomen. Although the authors do state that these procedures are to be performed only by experienced surgeons, it is arguable that it would have been appropriate to place more focus on the postoperative management of procedures in which the intensivist would be most expected to participate. Regarding the chapter on epidural analgesia, one would not expect most intensivists to perform this procedure unless they had prior anesthesiology training. Therefore, a detailed explanation of the procedure might not have been required. That said, the related section on anticoagulation was useful because careful coordination between the intensive care team (managing anticoagulation) and the 
acute pain service (usually managing any neuraxial analgesia) is required for safe regional anesthesia management.

The general flow of information and ease of readability of the text did present some limitations, as there are several grammatical errors throughout the manual. Although most were minor and the authors' points were still conveyed to the reader, the frequency with which they occurred was somewhat distracting and has the potential to affect comprehension. Notably, there was an occasional serious grammatical mistake that compromised the clarity of the text. Before attempting any procedure, I believe it is imperative that the learner clearly understands all relevant anatomy, the steps involved, and complications that may occur. Therefore, a manual whose goal is to provide procedural guidance should be clear, concise, and devoid of potentially confusing grammatical mistakes.

Although Dr. Gurjar sought a structured template for each chapter, with appropriate headings and subheadings, the organization of information within chapters was occasionally confusing. For example, the chapter on laryngeal mask airway insertion reviews alternate methods of insertion under the heading of Technique and
Equipment prior to the description of the standard technique, which is presented under the heading of Procedure many pages later. The chapter on thoracentesis refers to "elsewhere" in the manual for the applied anatomy of the intercostal space, which is delineated in a subsequent chapter on tube thoracostomy. Other chapters were somewhat repetitive, with identical information being repeated under multiple subheadings.

To conclude, Dr. Gurjar's Manual of ICU Procedures is a comprehensive text, with detailed explanations of many intensive care procedures. It includes procedures commonly performed by the intensivist as well as relevant critical care procedures often performed by other physicians and members of the interdisciplinary team. With improved editorial direction in a possible second edition, this manual has the potential to be a worthwhile resource for any physician practicing intensive care medicine.

Conflicts of interest None declared.

Editorial responsibility This submission was handled by Dr. Hilary P. Grocott, Editor-in-Chief, Canadian Journal of Anesthesia. 